\title{
Ballistic collective group delay and its Goos-Hänchen component in graphene
}

\author{
$\mathrm{Yu}$ Song $^{1, *}$ and Han-Chun $\mathrm{Wu}^{2}$ \\ ${ }^{1}$ Department of Physics and State Key Laboratory of Low-Dimensional Quantum Physics, \\ Tsinghua University, Beijing 100084, People's Republic of China \\ ${ }^{2}$ School of Physics and CRANN, Trinity College Dublin, Dublin 2, Ireland
}

(Dated: July 6, 2018)

\begin{abstract}
We theoretically investigate the experimental observable of the ballistic collective group delay (CGD) of all the particles on the Fermi surface in graphene. First, we reveal that, lateral GoosHänchen $(\mathrm{GH})$ shifts along barrier interfaces contribute an inherent component in the individual group delay (IGD). Then, by linking the complete IGD to spin precession through a dwell time, we suggest that, the CGD and its GH component can be electrostatically measured by a conductance difference in a spin-precession experiment under weak magnetic fields. Such an approach is feasible for almost arbitrary Fermi energy. We also indicate that, it is a generally nonzero self-interference delay that relates the IGD and dwell time in graphene.
\end{abstract}

\section{INTRODUCTION}

It is well known that the process of a particle quantum tunneling through a barrier will take a time duration. Among various proposed expressions [1, 2], group delay $[3,4]\left(\tau_{g}\right.$, also known as phase time in the literature) and dwell time $[4,5]\left(\tau_{d}\right)$ are two well-established ones. The group delay is the duration between the appearing time of the reflection or transmission particle pulse and the arrival time of the incident pulse, while the dwell time is the time a particle lingers in the barrier region (see, Fig. 1b). They describe the tunneling speed through a barrier in different aspects, and are both of paramount importance for solid-state devices working at high frequencies [6]. Recently, as graphene rises as a star material in condensed matter physics, extensive efforts [7-13] have been devoted to the investigations of group delay and/or dwell time in it.

Unlike in single-electron devices, the current in bulk graphene devices is contributed by numerous electrons or holes on half of the Fermi surface. This fact implies that a collective group delay (CGD) of all these particles rather than an individual group delay (IGD) of a single particle should be adopted to evaluate the tunneling speed in graphene. The CGD can be defined as the summation of mode $(n)$-dependent IGDs weighted with corresponding transmission probability $\left(T_{n}\right)$, i.e., $\tau_{g}^{C} \equiv \Sigma_{n} T_{n} \tau_{g}^{(n)}$. In this work we theoretically investigate the CGD in graphene. Our motivation is two-fold. (i) CGD in graphene is not directly observable. Very recently, an approach using a Larmor clock $[5,14]$ has been suggested to measure the transmission times $\tau^{(p)}$ $\left(p=1,2, \tau^{(1)}=\tau_{g}^{C} / \Sigma_{n} T_{n}\right)$ at the especial Fermi energy that aligns to the barrier charge neutrality point (or Dirac point) [8]. However, the tunability of the Fermi energy is necessary in graphene devices; while the dynamics in graphene becomes totally different when the Fermi energy aligns to or diverges from the barrier Dirac

\footnotetext{
*kwungyusung@gmail.com
}

(a)

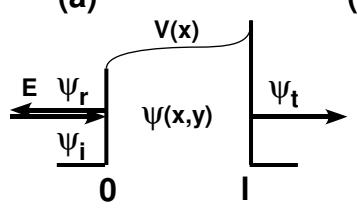

(b)
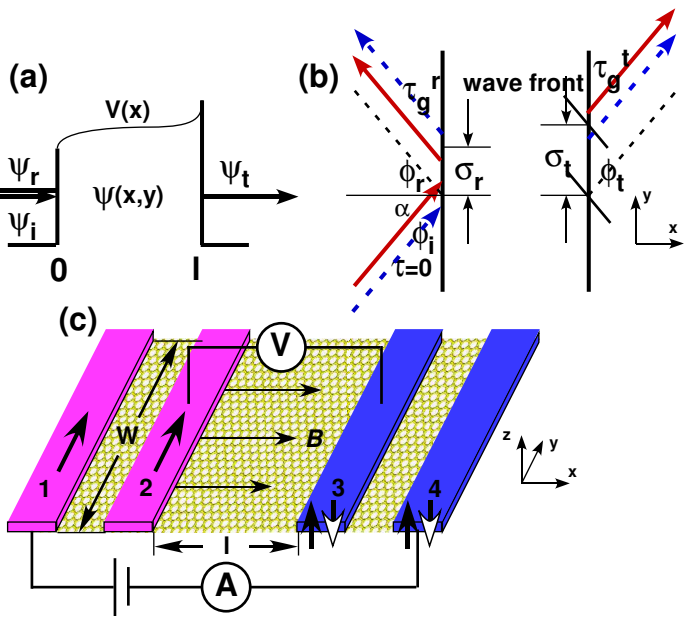

FIG. 1. (color online) (a) Sectional and (b) top schematic views for a particle quantum tunneling through a potential barrier in graphene. The red solid and blue dashed lines respectively stand for the ballistic trajectories of the $A$ and $B$ components, which are located at $\pm 1 / 2 k_{x}, \sigma_{r} \mp 1 / 2 k_{x}$, and $\sigma_{t} \pm 1 / 2 k_{x}$ for the incident, reflected, and transmitted beams, respectively. (c) Experimental setup for measuring the CGD by spin precession. Four ferromagnetic electrodes magnetized respectively along the $y_{-}, y_{-}, z(\bar{z})$-, and $z(\bar{z})$-axis are contacted to the graphene barrier, and a weak magnetic field is applied in the graphene plane along the $x$-axis. The contact induced potential [22] can be avoided by such four points probe [23] and the intrinsic conductance through the barrier can be measured by $I_{14} /\left.V_{23}\right|_{V_{23} \rightarrow 0}$. The length of the electrodes themselves are assumed to be small compared with $l$ so that the precession in them can be neglected.

point (i.e., pseudodiffusive [8] for the former and ballistic $[15,16]$ for the latter case). So, how to measure the ballistic CGD at arbitrary Fermi energy is still a basic problem. To propose a feasible approach to measure the ballistic CGD at arbitrary Fermi energy is our first motivation. (ii) The ballistic transport in graphene is essentially two dimensional (2D). As a result, a lateral Goos-Hänchen (GH) shift [17] occurs for the reflected or transmitted wave packet along the corresponding inter- 
face [18-21] (see Fig. 1b). As we will show, such GH shift makes an intrinsic contribution to the IGD. This fact has not been noticed in previous studies in graphene. It should be clarified no matter for the sake of itself or before we consider the measure problem of CGD. To obtain the complete expression of 2D IGD with the GH component contained is our second motivation.

In this work, through the analogy between ballistic electrons and photons, we obtain the complete expression of the 2D IGD and show that, the inherent GH component adds an asymmetric feature to the IGD's energy dependence. By linking the 2D IGD to spin precession through the 2D dwell time, we further suggest that, for any Fermi energy comparable with the barrier height, the CGD and its GH component can be probed through conductance measurements in a spin precession experiment under weak magnetic fields.

\section{INHERENT GH COMPONENT IN 2D IGD}

To investigate the 2D IGD, let us consider the 2D quantum tunneling through a potential barrier in graphene (see, Fig. 1(a)(b)). The size of the graphene sample is set to be smaller than the electron mean free path $(\ell \sim$ $0.5-1 \mu \mathrm{m}[15,16])$ and the phase-relaxation length $\left(L_{\phi} \sim\right.$ $3-5 \mu \mathrm{m}[15])$ to ensure the system stays in the ballistic coherent regime. A real potential occupies the region of $0<x<l$ and is translational invariant in the $y$-direction; it can be induced by a top gate due to the electric field effect [24]. The Fermi energy is $E$ and can be controlled by a back gate [24]. The sample width $W$ in the $y$-direction is several times of $l$ to ensure that the edge details are not important [25].

An electron with a central incident angle of $\alpha \in$ $(-\pi / 2, \pi / 2)$ at the Fermi surface incidents from the left side of the barrier. In the stationary state description, the spinor of electron envelope function $\Psi=\left(\psi_{A}, \psi_{B}\right)^{T}$ can be represented as a wave packet of a weighted superposition of plane wave spinors (each being a solution of Dirac's equation) $[18,21]$. The appearing loci $\left(\sigma_{\xi}^{\eta}\right)$ and moments $\left(\tau_{g}^{\xi \eta}\right)$ for the $A$ and $B(\eta= \pm)$ component of the incident, reflection, and transmission $(\xi=i, r, t)$ packet peaks (see, Fig. 1(b)) are determined by the following condition: the gradient of the total phase $\left(\phi_{T}^{\xi \eta}=\phi_{\xi}+k_{y} \sigma_{\xi}^{\eta}-E \tau_{g}^{\xi \eta} / \hbar\right)$ in the wave vector $\left(\boldsymbol{k}=k_{x} \hat{x}+k_{y} \hat{y}\right)$ space must vanish. Here, $r=|r| e^{i \phi_{r}}$ $\left(t=|t| e^{i \bar{\phi}_{t}}\right)$ and $\phi_{r}\left(\phi_{t} \equiv \bar{\phi}_{t}+k_{x} l\right)$ are the reflection (transmission) coefficient and corresponding phase shift, respectively (see, Fig. 1(b)). This is similar to the optical case [26] but with the extra sublattice degree of free$\operatorname{dom}(\eta)$. Since $k_{x}=E \cos \alpha / \hbar v_{F}$ and $k_{y}=E \sin \alpha / \hbar v_{F}$, the above condition means two independent conditions $\partial \phi_{T}^{\xi \eta} / \partial E=0$ and $\partial \phi_{T}^{\xi \eta} / \partial k_{y}=0 . \quad$ A comparison of the second conditions between the reflection (transmission) and incident beams gives the sublattice dependent lateral GH shift $[18,21]: \sigma_{r}^{ \pm}=-\left(\partial \phi_{r} / \partial k_{y}\right)_{E} \mp 1 / k_{x}$ and

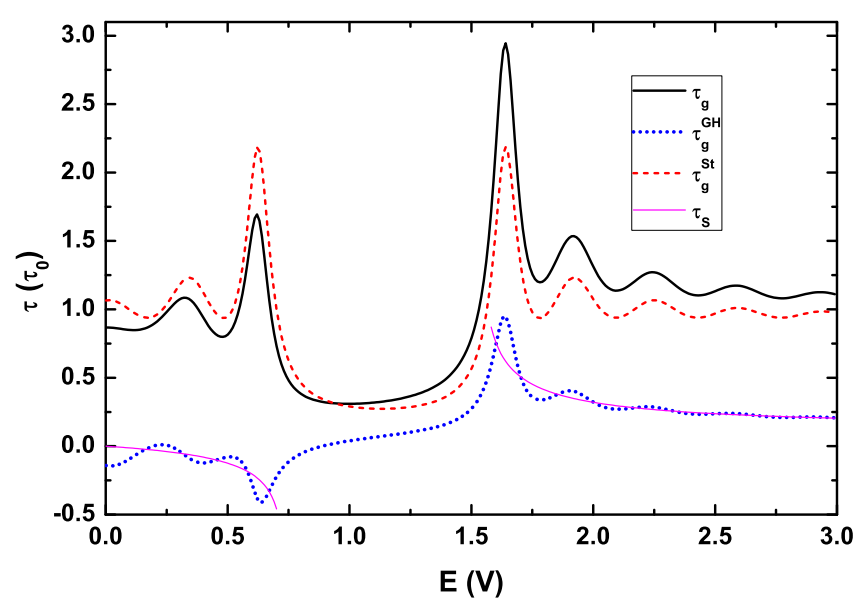

FIG. 2. (color online) The 2D IGD, its scattering, and GH components (in units of the equal time $\tau_{0} \equiv l / v_{F}$ ) as a function of the Fermi energy at $\alpha=20^{\circ}$. The magenta thin solid curve stands for the time associated with the Snell shift, $\tau_{S}=\sigma_{S} \sin \alpha / v_{F}$. The parameters of the potential barrier (which are also used in Figs. 3-5) are $l / l_{0}=1$ and $V / E_{0}=3 \pi$ with $l_{0}$ and $E_{0} \equiv \hbar v_{F} / l_{0}$ being a length unit and energy unit, respectively.

$\sigma_{t}^{ \pm}=-\left(\partial \phi_{t} / \partial k_{y}\right)_{E}$ (see, Fig. 1(b)). A comparison of the first conditions gives the reflection and transmission 2D IGD,

$$
\tau_{g}^{\xi}=\hbar\left[\frac{\partial\left(\phi_{\xi}+k_{y} \sigma_{\xi}\right)}{\partial E}\right]_{\alpha} .
$$

Here sublattice independent average values of GH shifts $\sigma_{\xi}=-\left(\partial \phi_{\xi} / \partial k_{y}\right)_{E}$ have been used to evaluate the IGD for the electron spinor rather than its sublattice components. For asymmetric barriers, there is a difference between the reflection and transmission in both $\phi_{\xi}$ and $\sigma_{\xi}$, so a bidirectional 2D IGD can be defined as $\tau_{g}=\sum_{\xi}|\xi|^{2} \tau_{g}^{\xi}$.

In the $1 \mathrm{D}$ case the bidirectional IGD has an expression of $\tau_{g}^{S t}=\sum_{\xi}|\xi|^{2} \hbar d \phi_{\xi} / d E$, which stems solely from the phase shifts introduced by scattering at the interfaces [27]. Comparing the two expressions, we can find that the differences come in two aspects between the $2 \mathrm{D}$ and $1 \mathrm{D}$ cases. Firstly, the differential in the $1 \mathrm{D}$ case becomes partial differential in the $2 \mathrm{D}$ one, i.e., $d / d E \rightarrow(\partial / \partial E)_{\alpha}$. Secondly, besides the scattering phase shifts in the 1D case, the GH shifts also contribute inherent phase shifts in the $2 \mathrm{D}$ case, i.e., $\phi_{\xi} \rightarrow \phi_{\xi}+k_{y} \sigma_{\xi}$.

To highlight the intrinsic contribution of the GH shifts, we rewrite the bidirectional 2D IGD as

$$
\tau_{g}=\tau_{g}^{S t}+\tau_{g}^{G H}
$$

The first component $\tau_{g}^{S t}=\sum_{\xi}|\xi|^{2} \hbar\left(\partial \phi_{\xi} / \partial E\right)_{\alpha}$ comes from the scattering phase shifts at the interfaces, which is the same as the 1D case; while the second component $\tau_{g}^{G H}=\sum_{\xi}|\xi|^{2} \sigma_{\xi} \sin \alpha / v_{F}$ results from the $\mathrm{GH}$ shifts, which is totally absent in the $1 \mathrm{D}$ case. Note, we have 
used the relation $\hbar\left(\partial k_{y} / \partial E\right)_{\alpha}=\sin \alpha / v_{F}$ to get the $\mathrm{GH}$ component, since the GH shifts are not explicit functions of the energy. We would like to stress that, although the GH component displays a partial differential respective to $k_{y}$ at fixed $E$ (i.e., $\tau_{g}^{G H}=\sum_{\xi}|\xi|^{2} \sigma_{\xi} \sin \alpha / v_{F}$ and $\left.\sigma_{\xi}=-\left(\partial \phi_{\xi} / \partial k_{y}\right)_{E}\right)$, this component essentially comes from the condition that the energy gradient of the GH phase shifts (i.e., $\partial / \partial E$ ) must vanish. We can understand the GH component as following. At time $\hbar\left(\partial \phi_{t} / \partial E\right)_{\alpha}$, the wave front of the transmitted beam reaches $(l, 0)$ and then propagates freely with a velocity of $v_{F}$ to the final position $\left(l, \sigma_{t}\right)$ (see, Fig. 1(b)). This step will cost a duration of $\sigma_{t} \sin \alpha / v_{F}$ since the wave front is perpendicular to the propagation direction. This picture also holds for the reflected beam. A weighted average of the transmission and reflection gives $\tau_{g}^{G H}$.

Figure 2 shows clearly the contribution of the GH component to the 2D IGD. One can see that $\tau_{g}^{S t}$ is symmetric about the center $\left(E / V=\cos ^{-2} \alpha\right)$ of the transmission gap (TG) due to the symmetry of $\phi_{\xi}$ about it. On the contrary, $\tau_{g}^{G H}$ stemming from the quantum GH shifts is asymmetric about the TG's center. The quantum GH shift displays the same trend as the classical shift predicted by the Snell's law $\left(\sigma_{S}=l \tan \beta\right.$ with $\beta=\sin ^{-1}\left(\hbar v_{F} k_{y} /(E-V)\right)$ being the refracted angle). The latter is obviously asymmetric as it is negative (positive) in the low (high) energy range (see Fig. 2). In total, the GH component not only quantitatively contributes a part of order of $\sigma_{S} \sin \alpha / v_{F}$ to the 2D IGD, but also qualitatively results in the remarkable asymmetric feature in the energy dependence of the 2D IGD.

\section{MEASURING THE CGD BY SPIN PRECESSION-INDUCED CONDUCTANCE DIFFERENCE}

Having obtained the complete expression for the IGD, we now seek physical observable for the CGD at arbitrary Fermi energy. The Larmor precession of the electron spin in a magnetic field provides a clock for studying the electron dynamics $[5,8,28]$. Here, we consider a configuration where the magnetic field is applied in the graphene plane along the $x$-axis (see, Fig. 1(c)). In such a configuration, the dynamical perturbation by the Lorentz force is avoided, and the only effect of the magnetic field is to cause spin precession round the $x$-axis. For electrons with spins initially directing the $y$-axis, a duration of this precession leads to different transmission probabilities in the $z$ and $\bar{z}$ directions. We calculate the spindependent transmission probability $\left(T_{z(\bar{z}) y}=\left|t_{z(\bar{z}) y}\right|^{2}\right)$ in a pseudospin-spin direct product space (a similar method is used in Ref. [8]), where $T_{z(\bar{z}) y}$ denotes the transmission probability for an electron incident from electrode 2 (with $y$-directed spin) and transmitted to electrode 3 (with $z$ or $\bar{z}$-directed spin) (see, Fig. 1(c)). An explicit equality between the spin polarization in transmission probabilities $P \equiv\left(T_{z y}-T_{\bar{z} y}\right) /\left(T_{z y}+T_{\bar{z} y}\right)$ and the dwell time

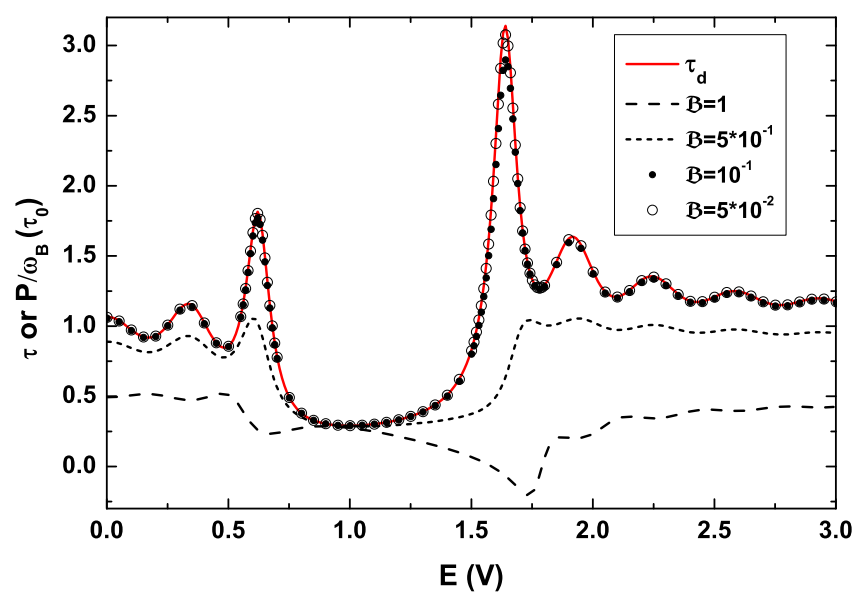

FIG. 3. (color online) The dwell time and $P / \omega_{B}$ at various $\mathcal{B}$ as a function of the Fermi energy at $\alpha=20^{\circ}$.

$\left(\tau_{d}=\int_{0}^{l}|\psi(x)|^{2} d x /\left(v_{F} \cos \alpha\right)\right)$ is found in the weak-field limit:

$$
\tau_{d}=\left.\left(P / \omega_{B}\right)\right|_{\mathcal{B} \rightarrow 0},
$$

where, $\omega_{B}=g \mu_{B} B$ is the Larmor frequency, $g$ is the gyromagnetic factor in graphene, $\mu_{B}$ is the Bohr magneton, and $B$ is the magnetic field with a reduced strength $\mathcal{B} \equiv \hbar \omega_{B} / 2 E_{0}$. This equality is clearly demonstrated in Fig. 3. Note, this equality is restricted in symmetric structures [29]. Under such restriction Eq. (3) holds for arbitrary incident energies and angles and can be interpreted physically in following. Rewriting the equality as $\omega_{B} \tau_{d}=P$ and multiplying both sides by $\hbar / 2$, the right hand side gives the expectation value of $S_{z}$ of the transmitted electrons. Such expectation value is determined by the product of the spin precession frequencey (i.e., the Larmor frequency) and the time the precession persists (i.e., the dwell time). This is just what the left hand side expresses. It is noted that Eq. (3) also holds in systems with parabolic dispersion relations and scale envelope functions [5].

To obtain the summation of the dwell time on half of the Fermi surface, we multiply Eq. (3) by $\left(T_{z y}+T_{\bar{z} y}\right) / 2$ (which tends to $T$ when $\mathcal{B} \rightarrow 0$ ). Then the right hand side of Eq. (3) can be further related to an experimental observable, the conductance $(G)$. This is because the conductance is defined as $G(E)=G_{0} \sum_{n} T_{n}$, where $G_{0}=2 e^{2} / h$ is the quantum conductance considering the twofold valley degeneracy. Thus, Eq. (3) can be rewritten as

$$
\sum_{n} \tau_{d}^{(n)} T_{n}=\left.\frac{G_{z y}(E)-G_{\bar{z} y}(E)}{2 \omega_{B} G_{0}}\right|_{\mathcal{B} \rightarrow 0},
$$

where $G_{z(\bar{z}) y}$ can be probed by $I_{14} /\left.V_{23}\right|_{V_{23} \rightarrow 0}$ in the proposed experimental setup (see, Fig. 1(c)).

We now try to link the CGD to the above conductance difference by the relation between the 2D IGD and the $2 \mathrm{D}$ dwell time. This relation can be obtained by making 


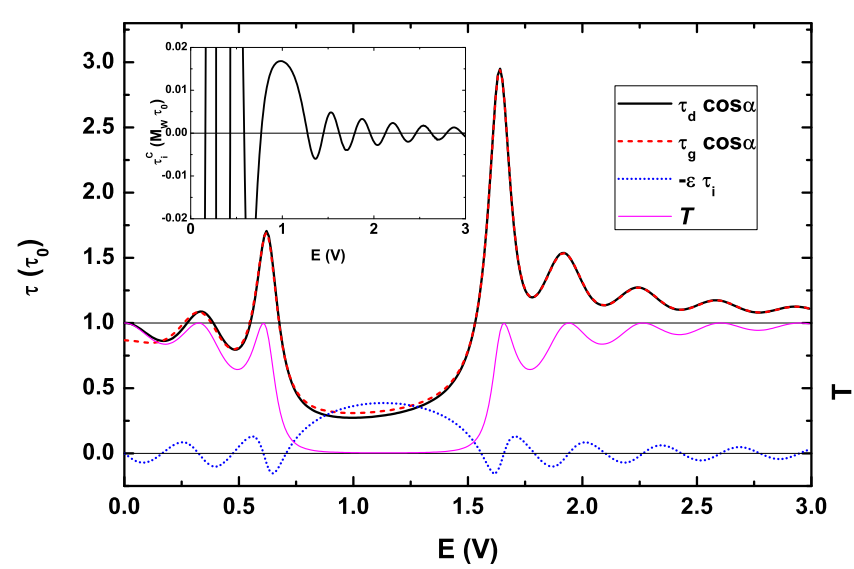

FIG. 4. (color online) The reduced IGD, dwell time, selfinterference delay, and transmission probability as a function of the Fermi energy at $\alpha=20^{\circ}$. Insert: the collective selfinterference delay versus the Fermi energy.

a variation of the time-independent Dirac equation at the barrier boundary with respect to the two individual variational parameters, $\left(k_{x}, k_{y}\right)$ or equivalently $(E, \alpha)$. The $2 \mathrm{D}$ feature of the tunneling process and the spinor nature of envelope functions should be taken into account in the variation, for which the detailed derivations can be found in the Appendix. We make the variation with respect to $E$ and $\sin \alpha$, since the variation results about them can be expediently related to $\tau_{g}^{S t}$ (see, Eqs. (A3)(A7) in the Appendix) and $\tau_{g}^{G H}$ (see, Eqs. (A8)-(A9) in the Appendix), respectively. The concise result for a rectangular barrier reads

$$
\tau_{d}=\tau_{g}+\tau_{i},
$$

where $\tau_{i}=\hbar[\operatorname{Re}(r) \cos \alpha+\operatorname{Im}(r) \sin \alpha] \sin \alpha /\left(E \cos ^{2} \alpha\right)$ is a self-interference delay (see, Eq. (A7) in the Appendix) stemming from the interference of the incident and reflection envelope functions in front of the barrier $[1,30]$. The correctness of this relation can be verified by numerically calculating and comparing the explicit expressions of $\tau_{d}, \tau_{g}$, and $\tau_{i}$.

Fig. 4 shows the 2D IGD, dwell time, and selfinterference delay in reduced form as a function of the Fermi energy at a fixed incident angle, where $\epsilon \equiv E / E_{0}$ is the reduction factor. One can see that the reduced selfinterference delay $\left(-\epsilon \tau_{i}\right)$ is symmetric about the TG's center; it achieves the maximum at the TG's center and oscillates around zero outside the TG. The selfinterference delay itself is important only in the low energy range (diverging as $E^{-1}$ when $E \rightarrow 0$ ). It disappears at (anti)resonant tunneling since there is no interference in front of the barrier. Accordingly, the IGD almost coincides with the dwell time except within the low energy range or around the TG's center.

The collective self-interference delay $\left(\tau_{i}^{C}\right)$ can be calculated by using $\sum_{n} T_{n}(E) \tau_{i}^{(n)}(E)=$ $M \int_{-\pi / 2}^{\pi / 2} \tau_{i}(E, \alpha) T(E, \alpha) d(\sin \alpha)$, where $M=$

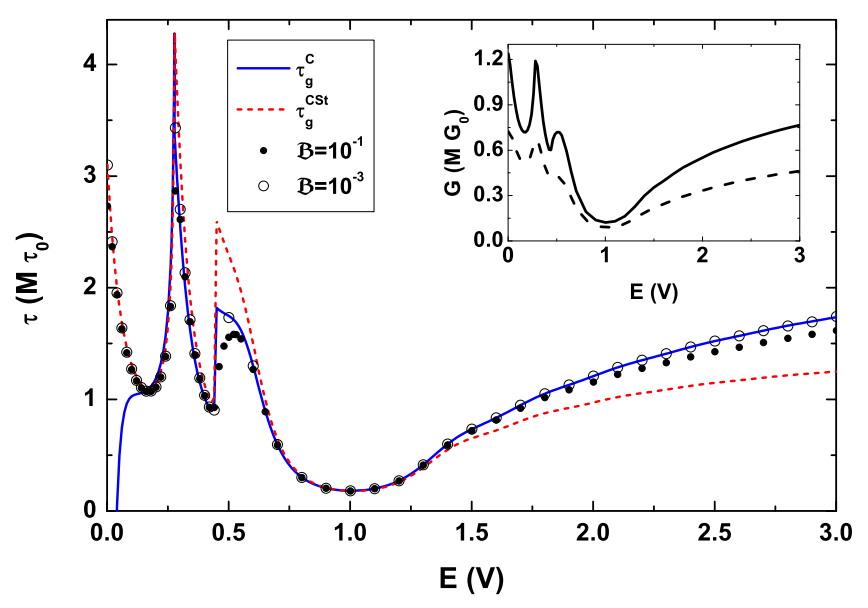

FIG. 5. (color online) The CGD, its scattering component, and the $\mathcal{B}$-dependent conductance difference (i.e., the right hand of Eq. (6)) as a function of the Fermi energy. Insert: $G_{z y}$ (solid) and $G_{\bar{z} y}$ (dashed) could be directly measured in the experiment for $\mathcal{B}=10^{-1}$.

$|E| W / h v_{F}=\left(|E| / E_{0}\right)\left(W / 2 \pi l_{0}\right) \equiv M_{E} M_{W}$ is the number of the transverse modes (which should be rather big). As can be seen in the insert of Fig. $4, \tau_{i}^{C}$ oscillates with $E$ with the amplitude disappearing exponentially at a relatively high Fermi energy. Therefore, Eqs. (4) and (5) imply that, for arbitrary Fermi energies comparable with $V$, the CGD can be directly measured by the spin-precession induced conductance difference under weak fields

$$
\left.\tau_{g}^{C}(E) \approx \frac{G_{z y}-G_{\bar{z} y}}{2 \omega_{B} G_{0}}\right|_{\mathcal{B} \rightarrow 0}
$$

Fig. 5 shows the CGD, its scattering component, and the magnetic field dependent conductance differences as a function of the Fermi energy. To clearly show the oscillation details in the low energy range, all these qualities are plotted for a single mode (i.e., divided by $M$ ). As is seen, the spin-precession induced conductance difference can be obtained by measuring $G_{z y}$ and $G_{\bar{z} y}$ separately (see insert in Fig. 5) and it increases for weaker $\mathcal{B}$ (see Fig. 5). At $\mathcal{B}=10^{-3}$, it is already a rather good measurement of the CGD for $E>0.2 \mathrm{~V}$. Thus, we provide an electrostatic approach to measure the CGD in graphene, which is feasible for almost arbitrary Fermi energy. This approach looks interesting when we notice that the CGD describes a dynamic process while the conductance is static.

In Fig. 5, it is also noted that, due to the GH shifts with different signs, the scattering component is always larger (smaller) than the expected value of the CGD when $E<V(E>V)$. Then, a comparison between the experimentally observed value of the conductance difference (the right hand side of Eq. (6)) and the theoretical prediction of the CGD (the left hand side of Eq. (6)) at Fermi energies away from the barrier Dirac point can be utilized to probe the inherent GH component of the 
CGD (or equivalently the intrinsic effect of the GH shifts on the CGD) in graphene.

\section{CONCLUSIONS AND REMARKS}

In summary, we have obtained the complete expression of the 2D IGD in graphene with the intrinsic contribution of the GH shifts being contained. Moreover, we have proposed an approach to probe the ballistic CGD and its inherent GH component by conductance measurements in a weak-field spin precession experiment. This approach is feasible for almost arbitrary Fermi energy. We have also indicated that, it is a generally nonzero self-interference delay that relates the 2D IGD and dwell time in graphene. The inherent $\mathrm{GH}$ component of the 2D IGD should also present in other 2D ballistic coherent electronic systems, since the derivations of it do not depend on the specific electronic excitation of graphene. The feasibility of the proposed approach to measure CGD in these systems is an interesting question.

\section{ACKNOWLEDGEMENT}

YS benefited from discussions with Prof. C. W. J. Beenakker and HCW was grateful to the SFI Short Term Travel fellowship support during his stay at PKU. This project was supported by the 973 Program of China (2011CB606405).
[1] E. H. Hauge and J. A. Støvneng, Rev. Mod. Phys. 61, 917 (1989).

[2] R. Landauer and Th. Martin, Rev. Mod. Phys. 66, 217 (1994).

[3] E. P. Wigner, Phys. Rev. 98, 145 (1955).

[4] F. T. Smith, Phys. Rev. 118, 349 (1960).

[5] M. Büttiker, Phys. Rev. B 27, 6178 (1983).

[6] H. Mizuta and T. Tanoue, The Physics and Applications of Resonant Tunneling Diodes (Cambridge University Press, Cambridge, 1995).

[7] A. E. Bernardini, Ann. Phys. 324, 1303 (2009).

[8] R. A. Sepkhanov, M. V. Medvedyeva, and C. W. J. Beenakker, Phys. Rev. B 80, 245433 (2009).

[9] Z. Wu, K. Chang, J. T. Liu, X. J. Li, and K. S. Chan, J. Appl. Phys. 105, 043702 (2009); D. Dragoman and M. Dragoman, ibid. 107, 054306 (2010).

[10] Y. Gong and Y. Guo, J. Appl. Phys. 106, 064317 (2009); X. G. Xu and J. C. Cao, Physica B 407, 281 (2012).

[11] M. Esmailpour, A. Esmailpour, R. Asgari, M. Elahi, and M. R. Rahimi Tabar, Solid State Commun. 150, 655 (2010).

[12] J. T. Liu, F. H. Su, H. Wang, and X. H. Deng, New J. Phys. 14, 013012 (2012).

[13] F. Sattari and E. Faizabadi, AIP Adv. 2, 012123 (2012); E. Faizabadi and F. Sattari, J. Appl. Phys. 111, 093724 (2012).

[14] N. Tombros, C. Jozsa, M. Popinciuc, H. T. Jonkman, and B. J. van Wees, Nature (London) 448, 571 (2007).

[15] F. Miao, S. Wijeratne, Y. Zhang, et al., Science, 317, 1530 (2007).

[16] X. Du, I. Skachko, A. Barker, et al., Nature Nano. 3, 491 (2008).

[17] This shift is named after the physicists who first observed it. See, F. Goos and H. Hänchen, Ann. Phys. (Leipzig) 436, 333 (1947).

[18] C. W. J. Beenakker, R. A. Sepkhanov, A. R. Akhmerov, and J. Tworzydło, Phys. Rev. Lett. 102, 146804 (2009).

[19] X. Chen, J. W. Tao, and Y. Ban, Euro. Phys. J. B 79, 203 (2011); M. Sharma and S. Ghosh, J. Phys.: Condens. Matter 23, 055501 (2011).

[20] Z. Wu, F. Zhai, F. M. Peeters, H. Q. Xu, and K. Chang, Phys. Rev. Lett. 106, 176802 (2011); F. Zhai, Y. Ma, and K. Chang, New J. Phys. 13, 083029 (2011).
[21] Y. Song, H. C. Wu, and Y. Guo, Appl. Phys. Lett. 100, 253116 (2012).

[22] Y. Wu, V. Perebeinos, Y. M. Lin, T. Low, F. Xia, and P. Avouris, Nano Lett. 12, 1417 (2012).

[23] B. Huard, J. Sulpizio, N. Stander, et al., Phys. Rev. Lett. 98, 236803 (2007)

[24] K. S. Novoselov, A. K. Geim, S. V. Morozov, D. Jiang, Y. Zhang, S. V. Dubonos, I. V. Grigorieva, and A. A. Firsov, Science 306, 666 (2004).

[25] J. Tworzydło, B. Trauzettel, M. Titov, A. Rycerz, and C. W. J. Beenakker, Phys. Rev. Lett. 96, 246802 (2006).

[26] A. M. Steinberg and R. Y. Chiao, Phys. Rev. A 49, 3283 (1994); C. F. Li, ibid. 65, 066101 (2002).

[27] H. G. Winful, Phys. Rev. Lett. 91, 260401 (2003).

[28] A. I. Baz', Sov. J. Nucl. Phys. 4, 182 (1967); ibid. 5, 161 (1967); V. F. Rybachenko, ibid. 5, 635 (1967).

[29] C. R. Leavens and G. C. Aers, Solid State Commun. 63, 1101 (1987).

[30] E. H. Hauge, J. P. Falck and T. A. Fjeldly, Phys. Rev. B 36, 4203 (1987).

[31] M. I. Katsnelson, K. S. Novoselov, and A. K. Geim, Nature Phys. 2, 620 (2006).

[32] X. L. Qi and S. C. Zhang, Rev. Mod. Phys. 83, 1057 (2011).

\section{Appendix A: Derivation for the relation between 2D IGD and dwell time in graphene}

Let us begin with the single-particle Dirac equation that governs the low-energy excitation in graphene. In the barrier region it reads as $\left[v_{F} \boldsymbol{\sigma} \cdot \mathbf{p}+V(x)\right] \boldsymbol{\Psi}(x, y)=$ $E \boldsymbol{\Psi}(x, y)$, where the pseudospin matrix $\boldsymbol{\sigma}$ has components given by Pauli's matrices and $\mathbf{p}=\left(p_{x}, p_{y}\right)$ is the momentum operator. The eigenstates $\boldsymbol{\Psi}(x, y)$ are twocomponent spinors with each component being the envelope function at sublattice site $A / B$ of the graphene sheet. Due to the translational invariance along the $y$ axis, the envelope function can be separated as $\boldsymbol{\Psi}=$ $\left[\psi_{A}(x), \psi_{B}(x)\right]^{T} e^{i k_{y} y}$ with $k_{y}=E \sin \alpha / \hbar v_{F}$. The $A$ and $B$ components along the $x$-direction are related by a pair 
of coupling first-order equations

$$
\frac{\partial}{\partial x} \psi_{A, B}= \pm k_{y} \psi_{A, B}-\frac{E-V}{i \hbar v_{F}} \psi_{B, A}
$$

which imply a decoupled second-order equation for both the $A$ - and $B$-components

$$
\left[\frac{\partial^{2}}{\partial x^{2}}+\frac{(E-V)^{2}-E^{2} \sin ^{2} \alpha}{\left(\hbar v_{F}\right)^{2}}\right] \psi_{A, B}=0 \text {. }
$$

We carry out the energy-variational form and conjugate form of Eq. (A2) and upon integration over the length of the barrier we get

$$
\begin{aligned}
& \left.\left(\frac{\partial \psi_{A, B}}{\partial E} \frac{\partial \psi_{A, B}^{*}}{\partial x}-\psi_{A, B}^{*} \frac{\partial^{2} \psi_{A, B}}{\partial E \partial x}\right)_{\alpha}\right|_{x=0} ^{x=l} \\
& =\int_{0}^{l} \frac{2 E \cos ^{2} \alpha-2 V(x)}{\left(\hbar v_{F}\right)^{2}}\left|\psi_{A, B}\right|^{2} d x .
\end{aligned}
$$

It is found that when is evaluated by the envelope function outside (inside) the barrier, the left (right) part can be related to $\tau_{g}^{S t}\left(\tau_{d}\right)$. However, we should note that Eq. (A3) is only valid inside the barrier as the spatial derivative of $\psi_{A, B}$ are not continuous on the potential boundary. To overcome this dilemma, we express $\partial \psi_{A, B} / \partial x$ inside the barrier by Eq. (A1) and their conjugate form. Since $\psi_{A, B}$ themselves are continuous, envelope functions inside the barrier can be replaced by the corresponding ones outside the barrier. Then the left part of Eq. (A3) can be evaluated. For the $A$-component it reads as

$$
J+K+\cos \alpha\left(-i r+i r^{*}\right) / \hbar v_{F},
$$

and for the $B$-component, the result becomes

$$
J+K+\cos \alpha\left(i e^{-i 2 \alpha} r-i e^{i 2 \alpha} r^{*}\right) / \hbar v_{F},
$$

where $J=\frac{i E}{\hbar v_{F}}\left\{[B(0)-A(0)]|r|^{2} \phi_{r}^{\prime}+[B(l)-A(l)]|t|^{2} \phi_{t}^{\prime}\right\}$ and $K=\frac{E}{\hbar v_{F}}\left\{[B(0)-A(0)]|r \| r|^{\prime}+[B(l)-A(l)]|t||t|^{\prime}\right\}$. Here the relation of lossless barriers $|t|^{2}+|r|^{2}=1$ has been used and the notations are adopted as $O^{\prime} \equiv$ $(\partial O / \partial E)_{\alpha}, A(x)=\sin \alpha+i \lambda(x) e^{i \alpha}, B(x)=\sin \alpha-$ $i \lambda(x) e^{-i \alpha}$, and $\lambda(x)=1-V(x) / E$, a ratio of the kinetic energy inside and outside the barrier.

Since $\psi^{*} \psi=\psi_{A}^{*} \psi_{A}+\psi_{B}^{*} \psi_{B}$, the relation for each spinor component should be added to get the relation for the spinor, which at last reads

$$
\begin{aligned}
& \frac{\int_{0}^{l}\left[\lambda(x)-\sin ^{2} \alpha\right]|\psi(x)|^{2} d x}{v_{F} \cos \alpha} \\
= & \lambda(0)|r|^{2} \hbar \phi_{r}^{\prime}+\lambda(l)|t|^{2} \hbar \phi_{t}^{\prime}-i \lambda(0) \hbar|r||r|^{\prime}-i \lambda(l) \hbar|t||t|^{\prime} \\
& +\hbar \frac{[\operatorname{Re}(r) \cos \alpha+\operatorname{Im}(r) \sin \alpha] \sin \alpha}{k} \frac{\partial k}{\partial E},
\end{aligned}
$$

where $\operatorname{Re}(r)(\operatorname{Im}(r))$ is the real (imaginary) part of $r$. We can see that, this equation is a general result that relates the integral of the weighted probability density inside the barrier (left part) and the weighted energyvariational behavior outside the barrier (right part). It is noted that, the factor $\lambda(x)$ has a critical role in the relation.

To clearly relate the general result Eq. (A5) with both $\tau_{d}$ and $\tau_{g}^{S t}$, we consider a restricted condition that $\lambda(x)$ is a constant under the barrier (i.e., a rectangular barrier). Note this condition is not necessary for the common semiconductors case [27], a reflection of the spinor nature of graphene. Under such condition, the third and fourth terms on the right hand side of Eq. (A5) disappear due to the lossless condition of the barrier $|t||t|^{\prime}+|r||r|^{\prime}=0$, and Eq. (A5) can be rewritten in terms of $\tau_{d}$ and $\tau_{g}^{S t}$, i.e., as a sub-relation

$$
\tau_{d}\left(\lambda-\sin ^{2} \alpha\right)=\tau_{g}^{S t} \lambda+\tau_{i} \cos ^{2} \alpha,
$$

where a self-interference delay is found from the last term of Eq. (A5),

$$
\tau_{i}=\frac{\hbar[\operatorname{Re}(r) \cos \alpha+\operatorname{Im}(r) \sin \alpha] \sin \alpha}{E \cos ^{2} \alpha} .
$$

Graphene is two dimensional, which means there are two independent variation parameters, $k_{x}$ and $k_{y}$ or $E$ and $\sin \alpha$. We have made the variation about $E$, the variation of Eq. (A2) about $\sin \alpha$ reads

$$
\begin{aligned}
& \left.\left(\frac{\partial \psi_{A, B}}{\partial \sin \alpha} \frac{\partial \psi_{A, B}^{*}}{\partial x}-\psi_{A, B}^{*} \frac{\partial^{2} \psi_{A, B}}{\partial \sin \alpha \partial x}\right)_{E}\right|_{x=0} ^{x=l} \\
& =\int_{0}^{l} \frac{-2 E^{2} \sin \alpha}{\left(\hbar v_{F}\right)^{2}}\left|\psi_{A, B}\right|^{2} d x .
\end{aligned}
$$

Following a similar way as above, we straightforwardly obtain the sub-relation between $\tau_{d}$ and $\tau_{g}^{G H}$ under the same restricted condition of a constant $\lambda$. The subrelation reads

$$
\tau_{d} \sin ^{2} \alpha=\tau_{g}^{G H} \lambda+\tau_{i}\left(\lambda-\cos ^{2} \alpha\right) .
$$

Making a simple addition of the two sub-relations in Eqs. (A6) and (A9) and taking into account $\tau_{g}=\tau_{g}^{S t}+$ $\tau_{g}^{G H}$ (see, Eq. (2) in the main body), we finally get

$$
\tau_{d}=\tau_{g}+\tau_{i}
$$

The correctness of this relation and the sub-relations can be verified by numerically calculating and comparing the explicit expressions of the five times. It may be valuable to indicate that, many previous works [9-11] have also concerned such relation and they all have achieved a same conclusion that $\tau_{g}=\tau_{d}$ (actually they mean $\tau_{g}^{S t}=\tau_{d}$ since $\tau_{g}^{G H}$ is not included in their discussions). The results obtained here clearly indicate that, it is a selfinterference delay that relates the group delay and dwell time in graphene, similar to the common semiconductor case [27].

For the normal incident or 1D tunneling case $(\alpha=0)$, $\tau_{i}$ vanishes (see the factor $\sin \alpha$ in Eq. (A7)), since no 
reflected portion thus no interference happens in front of the barrier due to the Klein tunneling [31]. The relation and subrelations revealed in Eqs. (A10), (A6), and (A9) and the expression for the self-interference delay Eq.
(A7) also hold for the tunneling of massless Dirac particles in topological surface states [32], where the real electron spin rather than the sublattice structure in graphene provides the Dirac structure. 